\title{
The Night Sky Monitoring Network in Hong Kong
}

\author{
Chun S. J. Pun ${ }^{1}$, Chu W. So ${ }^{2}$ and Chung F. T. Wong ${ }^{3}$ \\ ${ }^{1}$ Department of Physics, The University of Hong Kong, \\ Pokfulam, Hong Kong \\ email: jcspun@hku.hk \\ ${ }^{2}$ email: socw@hku.hk \\ ${ }^{3}$ email: terryfai@hku.hk
}

\begin{abstract}
The Night Sky Monitoring Network is a project that aims to study the extent, distribution, and properties of the light pollution condition in the populous metropolis of Hong Kong. Continuous measurements of the Night Sky Brightness (NSB) at strategically chosen locations that cover a wide range of population density and land usage were made, with over 2.5 million NSB readings collected in 18 months up to June 2012. Results from the project are presented, with focus on the contrast between the urban and rural night sky profiles, and light pollution contributions from artificial lightings. This project is supported by the Environment and Conservation Fund of the Hong Kong SAR government (ECF 10/2009, ECF 1/2007).
\end{abstract}

Keywords. Measurement: night sky brightness, light pollution, atmospheric effects.

The positions of the 18 measuring stations (equiped with Sky Quality Meters - Lens Ethernet) are overlayed on the night sky image taken by a camera mounted on the International Space Station taken in 2003 in Figure 1 (left), clearly indicating the contrast between the urban (red) and rural (blue) night sky profiles. The long-term trend of the median NSB at selected stations are shown in Figure 1 (right). The observed monthly fluctuations of NSB at any particular site was found to be due mostly to seasonal variations in meteorological factors such as cloud and rain (Please refer to contribution by So et al. in this volume). On the other hand, the relative ranking of different sites remained steady, with the NSB of rural sites consistently darker than those in urban locations by 3-4 mag, under similar meteorological conditions. This clearly indicated the polluting effects of artificial lightings.
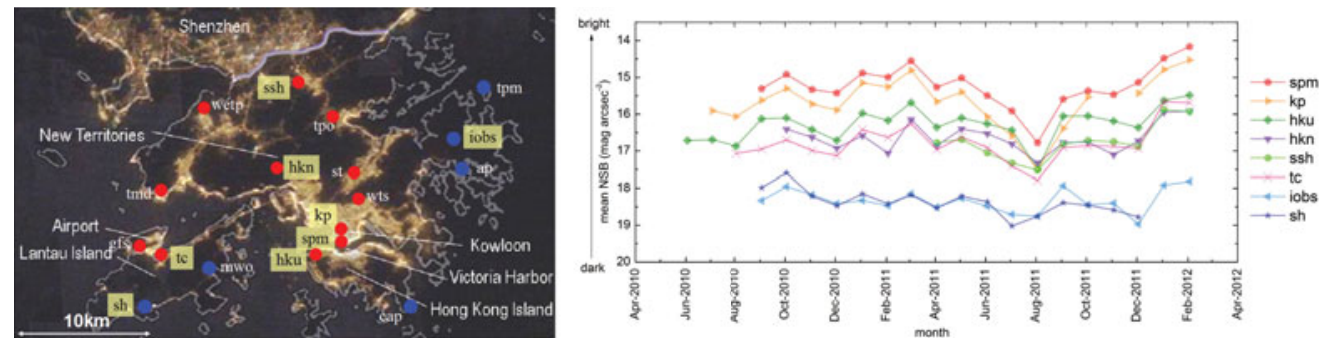

Figure 1. (Left) Locations of urban (red) and rural (blue) monitoring sites were overlaid on the night time picture of Hong Kong taken from the International Space Station; (Right) Monthly variations of the median NSB in selected sites.

For details, please refer to the project website: http://nightsky.physics.hku.hk 